\title{
ESTIMATES OF INVERSES OF MULTIVARIABLE TOEPLITZ MATRICES
}

\author{
HUGO J. WOERDEMAN
}

\begin{abstract}
The Gohberg-Semencul formula provides a formula for the inverse of a Toeplitz matrix based on the entries in the first and last columns of the inverse, under certain nonsingularity conditions. In this paper we study similar formulas for multivariable Toeplitz matrices, and we show that in the positive definite case these expressions provide upper bounds for the inverse in the Loewner order. Some numerical experiments regarding the proximity of the estimate are included.
\end{abstract}

Mathematics subject classification (2000): 15A09, 47B35, 65F30.

Keywords and phrases: multivariable Toeplitz, inverse formula, Gohberg-Semencul expressions.

\section{REFERENCES}

[1] Michael A. Dritschel And Hugo J. Woerdeman, Outer factorizations in one and several variables, Trans. Amer. Math. Soc., 357(11):4661-4679 (electronic), 2005.

[2] JefFrey S. Geronimo and Hugo J. Woerdeman, Positive extensions, Fejér-Riesz factorization and autoregressive filters in two variables, Ann. of Math. (2), 160(3):839-906, 2004.

[3] JEFFREY S. GERONIMO AND HUGO J. WOERDEMAN, The operator valued autoregressive filter problem and the suboptimal Nehari problem in two variables, Integral Equations Operator Theory, 53(3):343-361, 2005.

[4] I. GoHBERG AND V. OlshevsKy, Complexity of multiplication with vectors for structured matrices, Linear Algebra Appl., 202:163-192, 1994.

[5] I. C. GOHBERG AND G. HAĬNIG, Inversion of finite Toeplitz matrices consisting of elements of a noncommutative algebra, Rev. Roumaine Math. Pures Appl., 19:623-663, 1974.

[6] I. C. Gohberg AND A. A. SEMENCUl, The inversion of finite Toeplitz matrices and their continual analogues, Mat. Issled., 7(2(24)):201-223, 290, 1972.

[7] GEORG HEINIG AND KARLA Rost, Algebraic methods for Toeplitz-like matrices and operators, Akademie-Verlag, Berlin, 1984.

[8] Thomas Kailath, Sun Yuan Kung, And MarTin Morf, Displacement ranks of matrices and linear equations, J. Math. Anal. Appl., 68(2):395-407, 1979.

[9] VADIM OlsheVsKy, IVAN OSELEDETS, AND EugENE TyRTYSHNIKOV, Tensor properties of multilevel Toeplitz and related matrices, Linear Algebra Appl., 412(1):1-21, 2006.

[10] S. SERRA CAPIZZANO AND E. TYRTYSHNIKOV, Any circulant-like preconditioner for multilevel matrices is not superlinear, SIAM J. Matrix Anal. Appl., 21(2):431-439 (electronic), 1999.

[11] STEFANO SERRA CAPIZZANO, Matrix algebra preconditioners for multilevel Toeplitz matrices are not superlinear, Linear Algebra Appl., 343/344:303-319, 2002.

[12] William F. Trench, An algorithm for the inversion of finite Toeplitz matrices, J. Soc. Indust. Appl. Math., 12:515-522, 1964. 